International Journal of Pure and Applied Mathematics

Volume 105 No. 2 2015, 133-141

ISSN: 1311-8080 (printed version); ISSN: 1314-3395 (on-line version)

url: http://www.ijpam.eu

doi: http://dx.doi.org/10.12732/ijpam.v105i2.1

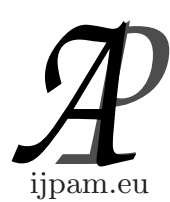

\title{
THE UNKNOTTING NUMBER OF SOME KNOTS
}

\author{
Moh'd Yasein $^{1 \S}$, Khaled Qazaqzeh ${ }^{2}$, Jenan Shtayat ${ }^{3}$ \\ ${ }^{1}$ Department of Mathematics \\ The Hashemite University \\ Zarqa, 13115, JORDAN \\ ${ }^{2,3}$ Department of Mathematics \\ Yarmouk University \\ Irbid, 21163, JORDAN
}

\begin{abstract}
We compute the unknotting number for infinite families of knots by using a famous inequality due to Murasugi that relates the unknotting number of a knot to the signature of the same knot. Also, we determine the unknotting number and show it is equal to two for some knots in the knot table with twelve crossings or less by another inequality due to Nakanishi that relates the unknotting number of a knot to the surgerical description number of the knot and by a theorem that is due to Kanenobu and Murakami.
\end{abstract}

AMS Subject Classification: 57M27, 57M26

Key Words: unknotting number, surgical view, knot signature

\section{Introduction}

For a classical knot $K$ in $S^{3}$, the unknotting number $u(K)$ is the minimum number of crossing changes required to deform the knot $K$ to the unknot where

Received: March 29, 2015

(C) 2015 Academic Publications, Ltd. url: www.acadpubl.eu

$\S_{\text {Correspondence author }}$ 
the minimum is taken over all regular projections of the knot $K$ [7, Page. 7]. The definition of this invariant is intuitive, but the dependence on the projections makes it very hard to compute. Even though some algebraic techniques are known to give a lower bound for $u(k)$, the unknotting number for many knots in the knot table with twelve crossings or less are still undetermined.

We use the following famous inequality due to Murasugi [8] that relates the unknotting number $u(K)$ to the signature $\sigma(K)$ of the knot $K$ to compute the unknotting number of infinite families of knots.

$$
0 \leq \frac{1}{2}|\sigma(K)| \leq u(K) .
$$

Also, we use the following inequality due to Nakanishi $[10,11]$ that relates the unknotting number $u(K)$ to the surgerical description number $(K)$ of the knot $K$ to determine the unknotting number of the knots $9_{37}, 10_{3}$ and $10_{20}$ and show it is equal to two.

$$
0 \leq(K) \leq u(K) .
$$

Finally, we use the following theorem to determine the unknotting number of the knots $12_{a_{802}}$ and $12_{a_{1166}}$ and show it is equal to two.

Theorem 1. Let $K$ be a nontrivial two-bridge knot. Then the following three conditions are equivalent.

1. $u(K)=1$.

2. There exist an odd positive integer $p>1$ and coprime, positive integers $m$ and $n$ with $2 m n=p \pm 1$ and $K$ is equivalent to $S\left(p, 2 n^{2}\right)$ in Schubert's notation as in [15].

3. $K$ can be expressed as $C\left(a, a_{1}, a_{2}, \ldots, a_{k}, \pm 2,-a_{k}, \ldots,-a_{2},-a_{1}\right)$ in Conway's normal form [4].

\section{Preliminaries and Results}

The knot signature was first defined by Trotter in [17] as the signature of some quadratic form defined in terms of an orientable surface spanning the knot and he showed that this signature is an invariant of the knot. Later on, the authors of [3] were able to define the knot signature in terms of the signature of any spanning surface. Therefore, they obtained an easier algorithm to compute this invariant, and it shows that this invariant can be computed combinatorially 


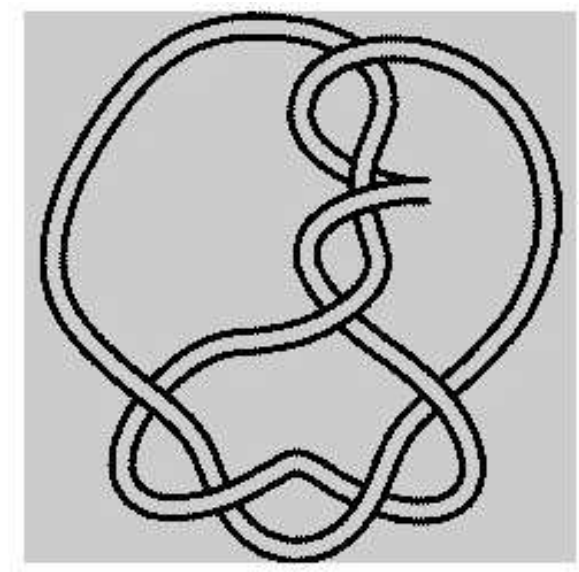

Figure 1: A diagram of the knot $K_{4}^{4}$

without going back to any spanning surface. We follow [9, Section. 2.1] for the computations of the signature through the paper. Murasugi in [8] relates the signature and the unknotting number of the knot $K$ by inequality 1 .

The surgical description of the knot $K$ appeared first in $[6,13,14]$, and then later Nakanishi obtained inequality 2 in $[10,11]$ that involves the surgery description number and the unknotting number of the knot $K$.

In this paper, we discuss three families of knots and we divide this section according to that.

\subsection{The Family $K_{n}^{m}$}

The knot $K_{4}^{4}$ is the knot with a diagram given in Figure ??. Now, we let $K_{n}^{m}$ be the knot with a similar diagram as the one in Figure ?? but with $n$ horizontal crossings and $m$ vertical crossings where $m$ is even to obtain a knot. It is clear that $u\left(K_{n}^{2}\right)=\left(K_{n}^{2}\right)=1$ by changing one of the vertical crossing.

Remark 2. It is a simple exercise using Reidemeister moves to show that $K_{n}^{m}$ is equivalent to $K_{m}^{n}$.

Proposition 3. We have

$$
\sigma\left(K_{n}^{m}\right)= \begin{cases}m, & \text { if } n \text { is odd } \\ 0, & \text { if } n \text { is even. }\end{cases}
$$

Proof. We color the regions of the diagram that represents the knot $K_{n}^{m}$ white and black such that the outside region is white to obtain $m+1$ of white 
regions. We number these regions from top to bottom starting with the outside region to be $R_{0}$. Therefore, the Goeritz matrix $G$ is the $m \times m$ given as follows:

$$
G=\left(\begin{array}{cccccc}
2 & -1 & 0 & \ldots & 0 & 0 \\
-1 & 2 & -1 & \ldots & 0 & 0 \\
\vdots & \vdots & \vdots & \ldots & \vdots & \vdots \\
0 & 0 & \ldots & -1 & 2 & -1 \\
0 & 0 & \ldots & 0 & -1 & n+1
\end{array}\right)
$$

We can take

$$
Q=\left(\begin{array}{cccccc}
\frac{1}{\overline{2}} & 0 & 0 & \ldots & 0 & 0 \\
\frac{1}{2} & \sqrt{\frac{2}{3}} & 0 & \ldots & 0 & 0 \\
\frac{1}{3} & \frac{2}{3} & \sqrt{\frac{3}{4}} & 0 & \ldots & 0 \\
\vdots & \vdots & \ldots & \vdots & \vdots & \vdots \\
\frac{1}{m-1} & \frac{2}{m-1} & \frac{3}{m-1} & \ldots & \sqrt{\frac{m-1}{m}} & 0 \\
\frac{1}{m} & \frac{2}{m} & \frac{3}{m} & \ldots & \frac{m-1}{m} & \sqrt{\frac{m}{n m+1}}
\end{array}\right) .
$$

So that

$$
Q G Q^{t}=I
$$

Therefore, we have $\operatorname{sign}(G)=m$ by Sylvester law [16]. Finally, the required result follows since we have

$$
\sigma\left(K_{n}^{m}\right)=\sigma(G)-\mu(D)=m-\left\{\begin{array}{ll}
0, & \text { if } n \text { is odd, } \\
m, & \text { if } n \text { is even, }
\end{array}= \begin{cases}m, & \text { if } n \text { is odd }, \\
0, & \text { if } n \text { is even }\end{cases}\right.
$$

Corollary 4. If $n$ is odd and $m$ is even, then $u\left(K_{n}^{m}\right)=\frac{m}{2}$.

Proof. It is clear that $u\left(K_{n}^{m}\right) \leq \frac{m}{2}$ since we can deform the diagram that represents the knot $K_{n}^{m}$ to a diagram of the unknot by changing the odd or the even $\frac{m}{2}$-vertical crossings. We also have $\frac{m}{2}$ is a lower bound of the $u\left(K_{n}^{m}\right)$ since $\left|\sigma\left(K_{n}^{m}\right)\right|=m$. Therefore, $u\left(K_{n}^{m}\right)=\frac{m}{2}$.

Lemma 5. We have $\operatorname{det}\left(K_{n}^{4}\right)=4 n+1$.

Proof. It follows since the knot $K_{n}^{4}$ is a two-bridge knot with Schubert's notation $S(4 n+1,4)$. 
Theorem 6. $u\left(K_{6}^{4}\right)=\left(K_{6}^{4}\right)=2$.

Proof. It is clear that $u\left(K_{6}^{4}\right) \leq 2$ by changing the two even or odd vertical crossings to obtain a diagram of the unknot. Now a single crossing change at a vertical crossing deforms the diagram of the knot $K_{6}^{4}$ to a diagram of the knot $K_{6}^{2}$. Suppose $\left(K_{6}^{4}\right)=1$. Therefore, by a surgical view $K_{6}^{2}$ has an Alexander matrix of the form

$$
\left(\begin{array}{cc} 
\pm \Delta_{K_{6}^{4}}(t) & r\left(t^{-1}\right) \\
r(t) & m(t)
\end{array}\right)
$$

where each entry is a Laurent polynomial in $t$ and the determinant is $\pm \Delta_{K_{6}^{2}}(t)$. Now, if we take $t=-1$, we obtain

$$
\left|\begin{array}{cc} 
\pm \Delta_{K_{6}^{4}}(-1) & r(-1) \\
r(-1) & m(-1)
\end{array}\right|= \pm \Delta_{K_{6}^{2}}(-1)= \pm 13 .
$$

From this equation, we obtain

$$
r^{2}(-1) \equiv \pm 13 \quad(\bmod 25) .
$$

This is a contradiction because for any integer $x$, we have

$$
x^{2} \equiv 0,1,4,6,9,11,14,16,19,21,24, \quad(\bmod 25) .
$$

So the above equation has no solution. Hence, we have $\left(K_{6}^{4}\right) \geq 2$. Finally, since $u\left(K_{6}^{4}\right) \leq 2$ and $2 \leq\left(K_{6}^{4}\right) \leq u\left(K_{6}^{4}\right)$ then $\left(K_{6}^{4}\right)=u\left(K_{6}^{4}\right)=2$.

Theorem 7. For the knot $K_{8}^{4}$, we have $u\left(K_{8}^{4}\right)=2$.

Proof. It is clear that $u\left(K_{8}^{4}\right) \leq 2$ by changing the two odd or even vertical crossings to obtain a diagram of the unknot. Also, it is an easy exercise to show that the knot $K_{8}^{4}$ is a two-bridge knot and it is given by $S(33,4)$ in Schubert's notation in [15]. Now suppose $u\left(K_{8}^{4}\right)=1$, then this knot is equivalent to $S\left(p, 2 n^{2}\right)$ in Schubert's notation as a result of the second part of Theorem ??. Hence $S(33,4)$ must be equivalent to $S\left(33,2 n^{2}\right)$, where $n=1,17$, or $2^{4}$. This is not the case since two knots $S(p, q)$, and $S\left(p, q^{\prime}\right)$ are equivalent in Schubert's notation iff $\pm q \equiv q^{\prime}$ or $\pm q q^{\prime} \equiv 1(\bmod p)$ as proved in [15]. Thus, we have $u\left(K_{8}^{4}\right)=2$.

We end this part of this subsection by this conjecture.

Conjecture 8. If $m$ and $n$ are even, then $u\left(K_{n}^{m}\right)=\min \left\{\frac{m}{2}, \frac{n}{2}\right\}$. 


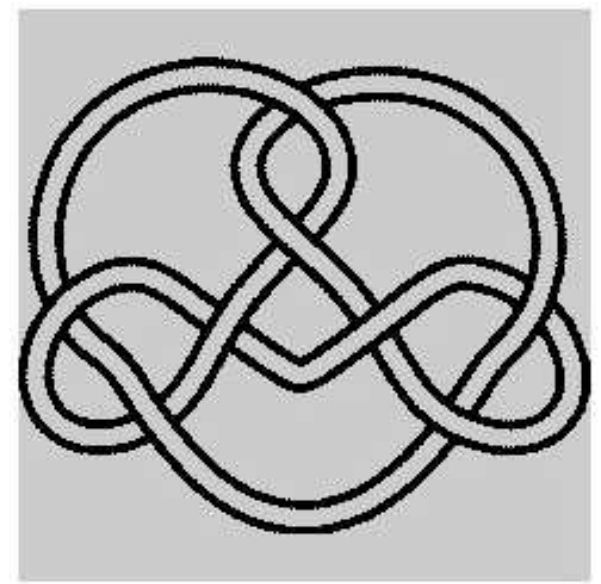

Figure 2: A diagram of the knot $T_{2}$

\subsection{The Family $T_{n}$}

The knot $T_{2}$ is the knot with a diagram given in Figure ??. Now, we let $T_{n}$ be the knot with a similar diagram as the one in Figure ?? but with $n$ vertical crossings.

Proposition 9. For any positive integer $n$,

$$
\sigma\left(T_{n}\right)= \begin{cases}4, & \text { if } n \text { is even } \\ 0, & \text { if } n \text { is odd }\end{cases}
$$

Proof. We color the regions of the diagram that represents the $\operatorname{knot} T_{n}$ white and black such that the outside region is black to obtain five white regions. We number these regions starting from the top right region to be $R_{0}$ and we keep going counterclockwise. Note that $R_{0}$ and $R_{1}$ are symmetric about the vertical axis the separates the knot into halves and similarly for $R_{2}$ and $R_{4}$. Therefore, the Goeritz matrix $G$ is the $4 \times 4$ given as follows:

$$
G=\left(\begin{array}{cccc}
n+2 & -1 & -1 & 0 \\
-1 & 2 & -1 & 0 \\
-1 & -1 & 4 & -1 \\
0 & 0 & -1 & 2
\end{array}\right)
$$

The principal minors are $n+2,2 n+3,7 n+6,12+27$. So by Sylvester's criterion $G$ is a positive definite matrix with 4 positive eigenvalues. Therefore, 
we have $\operatorname{sign}(G)=4$. Finally, the required result follows since we have

$$
\sigma\left(T_{n}\right)=\sigma(G)-\mu(D)=4-\left\{\begin{array}{ll}
0, & \text { if } n \text { is even, } \\
4, & \text { if } n \text { is odd, }
\end{array}= \begin{cases}4, & \text { if } n \text { is even } \\
0, & \text { if } n \text { is odd }\end{cases}\right.
$$

Corollary 10. For an even positive integer $n, u\left(T_{n}\right)=2$.

Proof. It is clear that $u\left(T_{n}\right) \leq 2$ by changing one of the left-side crossings and one of the right-side crossings to obtain a diagram of the unknot. Now, we know that 2 is a lower bound of $u\left(T_{n}\right)$ by the previous proposition. Therefore, we obtain $u\left(T_{n}\right)=2$.

Lemma 11. We have $\operatorname{det}\left(T_{n}\right)=12 n+9$ for $n \geq 1$.

Proof. It follows by induction on $n$ and applying [12, Lemma. 3.2] at any one of these $n$ vertical crossings.

Theorem 12. We have $u\left(T_{3}\right)=\left(T_{3}\right)=2$.

Proof. It is clear that $u\left(T_{n}\right) \leq 2$ by changing one of the left-side crossings and one of the right-side crossings to obtain a diagram of the unknot. A single crossing change at a vertical crossing deforms the diagram of the knot $T_{3}$ to a diagram of the knot $T_{1}$. Suppose $\left(T_{3}\right)=1$. Therefore, by a surgical view $T_{1}$ has an Alexander matrix of the form

$$
\left(\begin{array}{cc} 
\pm \Delta_{T_{3}}(t) & r\left(t^{-1}\right) \\
r(t) & m(t)
\end{array}\right)
$$

where each entry is a Laurent polynomial in $t$ and the determinant is $\pm \Delta_{T_{1}}(t)$. Now if we take $t=-1$, we obtain

$$
\left|\begin{array}{cc} 
\pm \Delta_{T_{3}}(-1) & r(-1) \\
r(-1) & m(-1)
\end{array}\right|= \pm \Delta_{T_{1}}(-1)= \pm 21 .
$$

From this equation, we obtain

$$
r^{2}(-1) \equiv \pm 21 \quad(\bmod 45)
$$

This is a contradiction because for any integer $x$, we have

$$
x^{2} \equiv 0,1,4,9,10,16,19,25,31,34,36,40 \quad(\bmod 45) .
$$

So the above equation has no solution. Hence, we have $\left(T_{3}\right) \geq 2$. Finally, since $u\left(T_{3}\right) \leq 2$ and $2 \leq\left(T_{3}\right) \leq u\left(T_{3}\right) \leq 2$ then $\left(T_{3}\right)=u\left(T_{3}\right)=2$. 


\begin{tabular}{|c|c|c|c|}
\hline Knot & Name & Unknotting number & Signature \\
\hline$K_{6}^{4}$ & $10_{3}$ & 2 & 0 \\
\hline$K_{8}^{4}$ & $12_{a_{1166}}$ & 2 & 0 \\
\hline$L_{3}^{5}$ & $10_{20}$ & 2 & -2 \\
\hline$L_{3}^{7}$ & $12_{a_{802}}$ & 2 & -2 \\
\hline$T_{3}$ & $9_{37}$ & 2 & 0 \\
\hline
\end{tabular}

Table 1: Knot table

Conjecture 13. For any odd integer $n \geq 5$, we have $=u\left(T_{n}\right)=2$.

In conclusion, we give a brief summary of the results of the main theorems in the given table and we provide the names of these knots in the knot table using [2].

\section{Acknowledgments}

The authors thanks the Abdus Salam International Center for Theoretical Physics for the kind hospitality during the course of this work. Finally, we are grateful to the authors of [1] for providing beautiful knot diagrams for some of the knots that have been used in this paper.

\section{References}

[1] D. Bar-Natan, S. Morrison, The Mathematica package Knot Theory, The Knot Atlas. http://katlas.math.toronto.edu/wiki/.

[2] J.C. Cha, C. Livingston, KnotInfo: Table of Knot Invariants, http://www.indiana.edu/ knotinfo, September 16, 2010.

[3] Gordon C. McA., R. A. Litherland, On the signature of a link, Invent. Math. 47 (1978), 53-69.

[4] K. Kawauchi, A Survey of Knot Theory, Birkhuäser Verlag, 1996.

[5] T. Kanenobu, H. Murakami, Two-bridge knots with unknotting number one, Proc. Amer. Math. Soc. 98 (1986), 499-502.

[6] J. Levine, A characterization of knot polynomials, Topology, 4 (1965), 135141. 
[7] R. Lickorish, An Introduction to Knot Theory, Springer-Verlag, 1997.

[8] K. Murasugi, On a certain numerical invariant of link types, Trans. Amer. Math. Soc., 117 (1965), 387-422.

[9] C. Manolescu, P. Ozsváth, On the Khovanov and knot Floer homologies of quasi-alternating links. In Proceedings of the 14th Gökova GeometryTopology Conference, International Press, Berlin, (2007), 60-81.

[10] Y. Nakanishi, A note on unknotting number, Math. Seminar Notes, Kobe Univ., 9 (1981), 99-108.

[11] Y. Nakanishi, A note on unknotting number, II, J. Knot Theory and its Ram., 14, No.1 (2005), 3-8.

[12] P. Ozsváth, Z. Szabó, On the Heegard Floer homology of branched doublecovers, Adv. Math., 194 (2005), 1-33.

[13] D. Rolfsen, A surgical view of Alexander's polynomial, In: Geometric Topology (Proc. Park City, 1974), Lecture Notes Math. 438 SpringerVerlag, (1974), 415-423.

[14] D. Rolfsen, Knots and links, Publish or Perish inc., 1976.

[15] H. Schubert, Knoten mit zwei Brücken, Math. Z., (1956), 133-170.

[16] J. Sylvester, A demonstration of the theorem that every homogeneous quadratic polynomial is reducible by real orthogonal substitutions to the form of a sum positive and negative squares, Philosophical Magazine, 4, No. 23 (1852), 138-142.

[17] H. Trotter, Homology of group systems with applications to knot theory, Ann. of Math., 2, No. 76 (1962), 464-498. 
